\title{
Event-Triggered Reliable Control in Networked Control Systems with Probabilistic Actuator Faults
}

\author{
Yuming Zhai, ${ }^{1,2}$ Ruixia Yan, ${ }^{3}$ Haifeng Liu, ${ }^{4}$ and Jinliang Liu ${ }^{5}$ \\ ${ }^{1}$ School of Economics and Management, Shanghai Institute of Technology, Shanghai 201418, China \\ ${ }^{2}$ Antai College of Economics \& Management, Shanghai Jiao Tong University, Shanghai 200052, China \\ ${ }^{3}$ School of Management, Shanghai University of Engineering Science, Shanghai 201620, China \\ ${ }^{4}$ College of Humanities, Donghua University, Shanghai 200051, China \\ ${ }^{5}$ Department of Applied Mathematics, Nanjing University of Finance and Economics, Nanjing, Jiangsu 210046, China
}

Correspondence should be addressed to Jinliang Liu; liujinliang2009@163.com

Received 25 December 2012; Accepted 4 March 2013

Academic Editor: Engang Tian

Copyright (C) 2013 Yuming Zhai et al. This is an open access article distributed under the Creative Commons Attribution License, which permits unrestricted use, distribution, and reproduction in any medium, provided the original work is properly cited.

\begin{abstract}
This paper introduces a novel event-triggered scheme into networked control systems which is used to determine when to transmit the newly sampled state information to the controller. Considering the effect of the network transmission delay and probabilistic actuator fault with different failure rates, a new actuator fault model is proposed under this event-triggered scheme. Then, criteria for the exponential mean square stability (EMSS) and criteria for codesigning both the feedback and the trigger parameters are derived by using Lyapunov functional method. These criteria are obtained in the form of linear matrix inequalities. A simulation example is employed to show that our event-triggered scheme can lead to a larger release period than some existing ones.
\end{abstract}

\section{Introduction}

Nowadays, more and more attention has been paid to the study of stability analysis and control design of networked control systems (NCSs). NCSs have a relatively new structure where the links from sensor to controller and from controller to actuator are not connected directly, but through a network $[1,2]$. The application of networks into control systems can be advantageous in terms of simplicity scalability, and costeffectiveness. However, the introduction of a communication network can also bring about many problems, such as network-induced delay and packet dropout. Therefore, the tasks in traditional systems, such as the control problems and signal estimation problem, should be reconsidered. In recent years, the stability analysis and control design for NCS have been invested, and lots of outstanding results have been obtained $[3,4]$. In these works, most are based on the periodic triggered control method, which is called a timetrigged control. In this triggering scheme, the fixed sampling period is determined under worst conditions such as external disturbances, uncertainties, and time-delays. However, in practical systems, the worst cases seldom occur. Thus, this kind of triggering method may often lead to transmitting many unnecessary signals through the network, which in turn will increase the load of network transmission and waste the network bandwidth. Hence, it is an important problem to reduce communication requirements.

Recently, event-triggered scheme for control design, advocating the use of actuation only when some function of the system state exceeds a threshold, has received considerable attention, and many important results have been reported [5-9]. Event triggering method provides a useful way of determining when the sampling action is carried out, compared with periodic sampling methods, and it has the following advantages: (1) it only samples when necessary; (2) the burden of the network communication is reduced; (3) the computation cost of the controller and the occupation of the sensor and actuator are reduced.

More specifically, a networked estimator problem for event-triggered sampling systems with packet dropouts was solved in [7]. Refrence[9] studied event design in eventtriggered feedback systems, and a novel event triggering 
scheme was presented to ensure exponential stability of the resulting sampled-data system. Refrence [8] studied how the event triggered as well as the self-triggered control systems could be reformed in the case of discrete-time systems. The methods for design or implication of controllers in the event-triggered form based on dissipation inequalities were proposed for both linear and nonlinear systems in [6]. The work in [5] examined event-triggered data transmission in distributed networked control systems with packet loss and transmission delays.

Moreover, in NCSs, the temporary measurements failure and probabilistic distortion are usually unavoidable for a variety of reasons, for example, networked delay, sensor/actuators aging, electromagnetic interference, and zero shift, which may lead to intolerable system performance [10]. Therefore, from a safety as well as performance point of view, it is required to design a reliable controller that can tolerate actuators failures as well as networked delay. Recently, the fault model has received a lot of interest, and lots of outstanding results have been obtained [11-13]. In [11], the authors considered the problem of delay-dependent adaptive reliable $H_{\infty}$ controller design against actuator faults for linear timevarying delay systems. By using a linear matrix inequality technique and an adaptive method, they established a new delay-dependent reliable $H_{\infty}$ controller, which guaranteed the stability and adaptive $H_{\infty}$ performance of closed-loop systems in normal and faulty cases. Considering the different failure rates of each sensor or actuator, the authors studied the reliable controller for networked control systems in [12,13], reliable controllers were designed, and sufficient conditions for the exponentially mean square stability of NCS were obtained. Up to now, to the best of authors' knowledge, there are no papers to deal with the reliable control for event-triggered networked control systems with probabilistic actuator faults, which still remains as a challenging problem.

In this paper, firstly, an new event-triggered scheme is introduced to networked control systems, which can reduce the burden of the network communication. Then, considering different failure rates of actuators and the measurements distortion of every actuator, a new probabilistic actuator fault model is proposed under the proposed event-triggered scheme. By using Lyapunov functional method, criteria for the exponential stability and criteria for codesigning both the feedback and the trigger parameters are derived in terms of linear matrix inequalities. A simulation example is given to show that the proposed event-triggered scheme is superior to some existing ones.

Notation. $\mathbb{R}^{n}$ and $\mathbb{R}^{n \times m}$ denote the $n$-dimensional Euclidean space and the set of $n \times m$ real matrices; the superscript " $T$ " stands for matrix transposition; $I$ is the identity matrix of appropriate dimension; $\|$. $\|$ stands for the Euclidean vector norm or the induced matrix 2-norm as appropriate; the notation $X>0$ (resp., $X \geq 0$ ) for $X \in \mathbb{R}^{n \times n}$ means that the matrix $X$ is real symmetric positive definite (resp., positive semi definite). When $x$ is a stochastic variable, $\mathbb{E}\{x\}$ stands for the expectation of $x$. For a matrix $B$ and two symmetric matrices $A$ and $C,\left[\begin{array}{ll}A & * \\ B & C\end{array}\right]$ denotes a symmetric matrix, where * denotes the entries implied by symmetry.

\section{System Description}

In this paper, we consider the following system:

$$
\dot{x}(t)=A x(t)+B u(t),
$$

where $x(t) \in \mathbb{R}^{n}$ and $u(t) \in \mathbb{R}^{m}$ denote the state vector and control vector, respectively; $A$ and $B$ are parameter matrices with appropriate dimensions.

Throughout this paper, we assume that the system (1) is controlled though a network.

As is well known, periodic sampling mechanism has been widely used in practical systems; however, it may often lead to transmitting many unnecessary signals through the network, which in turn increases the load of network transmission and wastes the network bandwidth. Therefore, for the control of networked control systems shown in Figure 1, in order to save network resources such as network bandwidth, it is significant to introduce an event triggered mechanism which decides whether the newly sampled state should be sent out to the controller. As is shown in Figure 1, the state are sampled regularly by the sampler of the smart sensor with period $h$ and feeds into an event generator that decides when to transmit the states to the controller via a network medium by a specified trigger condition, which will be given in sequel. The following function of network architecture in Figure 1 is expected.

(1) The states are sampled at time $k h$ by sampler with a given period $h$. The next state is at time $(k+1) h$.

(2) As is shown in Figure 1, the event generator is constructed between the sensor and the controller which is used to determine whether the newly sampled state will be sent out to the controller or not by using the following judgement algorithm:

$$
\begin{aligned}
& {[x((k+j) h)-x(k h)]^{T} \Omega[x((k+j) h)-x(k h)]} \\
& \leq \rho x^{T}((k+j) h) \Omega x((k+j) h) .
\end{aligned}
$$

(3) Under the event-triggered scheme (2), the release times are assumed to be $t_{0} h, t_{1} h, t_{2} h, \ldots$, where $t_{0}$ is the initial time. $s_{i} h=t_{i+1} h-t_{i} h$ denotes the release period of event generator in (2). Considering the effect of the transmission delay on the network system, we suppose that the time-varying delay in the network communication is $\tau_{k}$ and $\tau_{k} \in[0, \bar{\tau})$, where $\bar{\tau}$ is a positive real number. Therefore, the states $x\left(t_{0} h\right), x\left(t_{1} h\right), x\left(t_{2} h\right), \ldots$ will arrive at the controller side at the instants $t_{0} h+\tau_{0}, t_{1} h+\tau_{1}, t_{2} h+\tau_{2}, \ldots$, respectively. Notice that the set of the release instants, that is, $\left\{t_{0}, t_{1}, t_{2}, \ldots\right\}$ is a subset of $\{0,1,2, \ldots\}$. The amount of $\left\{t_{0}, t_{1}, t_{2}, \ldots\right\}$, depends on not only the value of $\rho$, but also the variation of the state. When $\rho=0,\left\{t_{0}, t_{1}, t_{2}, \ldots\right\}=\{0,1,2, \ldots\}$, it reduces to the case with periodic release times. 


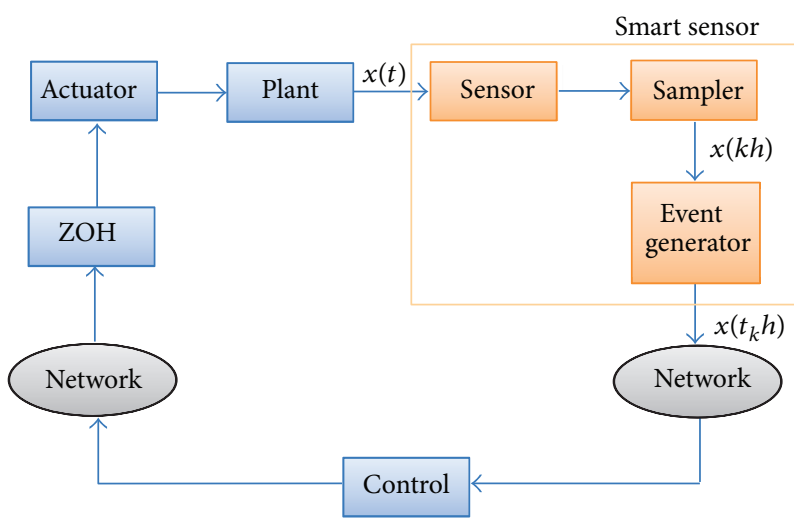

Figure 1: The structure of an event-triggered networked control system.

Based on the previous analysis, considering the effect of the transmission delay, the system model under the event generator with (2) can be described as

$$
\dot{x}(t)=A x(t)+B u\left(t_{k} h\right), \quad t \in\left[t_{k} h+\tau_{k}, t_{k+1} h+\tau_{k+1}\right) .
$$

Assumption 1. The actuators in the closed-loop systems have different failure rates because of different working conditions. Furthermore, the measurements distortion of every actuator is also take into consideration.

Under Assumption 1, the control $u(t)=K x(t)$ can be described as

$$
u^{F}(t)=\Xi K x(t)=\sum_{i=1}^{m} \xi_{i} C_{i} K x(t),
$$

where $\Xi=\operatorname{diag}\left\{\xi_{1}, \ldots, \xi_{m}\right\}$ and $\xi_{i}(i=1,2, \ldots, m)$ are $m$ unrelated variables taking values on the interval $[0, \theta]$, where $\theta \geq 1$, the mathematical expectation and variance of $\xi_{i}$ are $\mu_{i}$ and $\sigma_{i}^{2}(i=1,2, \ldots, m)$, and $C_{i}=\operatorname{diag}\{\underbrace{0, \ldots, 0}_{i-1}, 1, \underbrace{0, \ldots, 0}_{m-i}\}$.

Define $\bar{\Xi}=\operatorname{diag}\left\{\mu_{1}, \ldots, \mu_{m}\right\}=\sum_{i=1}^{m} \mu_{i} C_{i}$, and obviously, $\mathbb{E}(\Xi)=\bar{\Xi}, \mathbb{E}(\Xi-\bar{\Xi})=0, \mathbb{E}\left(\xi_{i}-\mu_{i}\right)^{2}=\sigma_{i}^{2}$.

Under (4), for $t \in\left[t_{k} h+\tau_{k}, t_{k+1} h+\tau_{k+1}\right)$, (3) can be rewritten as

$$
\dot{x}(t)=A x(t)+B \Xi K x\left(t_{k} h\right) .
$$

Remark 2. $u(t)$ is the control input without actuator failure, and $u^{F}(t)$ is the control input after actuator failures occur. $\xi_{i}=$ 0 and $\xi_{i}=1$ represent the meaning of completely failure or completely normal [14]. $0<\xi_{i}<1$ means partial failure [15, 16]. $\xi_{i}>1$ represents the condition of data distortion $[12,13]$.

Remark 3. $\mu_{i}=1$ does not represent that the $i$ th actuator is always in good work condition, but it means that the expectation of $i$ th actuator is 1 . cases.
Case 1. If $t_{k} h+h+\bar{\tau} \geq t_{k+1} h+\tau_{k+1}$, where $\bar{\tau}=\max \tau_{k}$, define a function $\tau(t)$ as

$$
\tau(t)=t-t_{k} h, \quad t \in\left[t_{k} h+\tau_{k}, t_{k+1} h+\tau_{k+1}\right) ;
$$

clearly,

$$
t_{k} \leq \tau(t) \leq\left(t_{k+1}-t_{k}\right) h+\tau_{k+1} \leq h+\bar{\tau} .
$$

Case 2. If $t_{k} h+h+\bar{\tau}<t_{k+1} h+\tau_{k+1}$, consider the following two intervals:

$$
\left[t_{k} h+\tau_{k}, t_{k} h+h+\bar{\tau}\right), \quad\left[t_{k} h+i h+\bar{\tau}, t_{k} h+i h+h+\bar{\tau}\right) .
$$

Since $\tau_{k} \leq \bar{\tau}$, it can be easily shown that there exists $d_{M}$ such that

$$
t_{k} h+d_{M} h+\bar{\tau}<t_{k+1} h+\tau_{k+1} \leq t_{k} h+d_{M} h+h+\bar{\tau} .
$$

Moreover, $x\left(t_{k} h\right)$ and $t_{k} h+i h$ with $i=1,2, \ldots, d_{M}$ satisfy (2). Let

$$
\begin{gathered}
I_{0}=\left[t_{k} h+\tau_{k}, t_{k} h+h+\bar{\tau}\right), \\
I_{i}=\left[t_{k} h+i h+\bar{\tau}, t_{k} h+i h+h+\bar{\tau}\right), \\
I_{d_{M}}=\left[t_{k} h+d_{M} h+\bar{\tau}, t_{k+1} h+\tau_{k+1}\right),
\end{gathered}
$$

where $i=1,2, \ldots, d_{M}-1$. One can see that

$$
\left[t_{k} h+\tau_{k}, t_{k+1} h+\tau_{k+1}\right)=\bigcup_{i=0}^{i=d_{M}} I_{i} .
$$

Define

$$
\tau(t)= \begin{cases}t-t_{k} h, & t \in I_{0}, \\ t-t_{k} h-i h, & t \in I_{i}, i=1,2, \ldots, d_{M}-1, \\ t-t_{k} h-d_{M} h, & t \in I_{d_{M}} .\end{cases}
$$

then, we have

$$
\begin{gathered}
t_{k} \leq \tau(t)<h+\bar{\tau}, \quad t \in I_{0}, \\
t_{k} \leq \bar{\tau} \leq \tau(t)<h+\bar{\tau}, \quad t \in I_{i}, \quad i=1,2, \ldots, d_{M}-1, \\
t_{k} \leq \bar{\tau} \leq \tau(t)<h+\bar{\tau}, \quad t \in I_{d_{M}},
\end{gathered}
$$

where the third row in (13) holds because $t_{k+1} h+\tau_{k+1} \leq t_{k} h+$ $\left(d_{M}+1\right) h+\bar{\tau}$. Obviously,

$$
\begin{aligned}
& 0 \leq \tau_{k} \leq \tau(t) \leq h+\bar{\tau} \triangleq \tau_{M}, \\
& t \in\left[t_{k} h+\tau_{k}, t_{k+1} h+\tau_{k+1}\right) .
\end{aligned}
$$

In Case 1, for $t \in\left[t_{k} h+\tau_{k}, t_{k+1} h+\tau_{k+1}\right)$, define $e_{k}(t)=0$. In Case 2, define

$$
e_{k}(t)= \begin{cases}0, & t \in I_{0}, \\ x\left(t_{k} h\right)-x\left(t_{k} h+i h\right), & t \in I_{i}, i=1,2, \ldots, d_{M}-1, \\ x\left(t_{k} h\right)-x\left(t_{k} h+d_{M} h\right), & t \in I_{d_{M}} .\end{cases}
$$


From the definition of $e_{k}(t)$ and the triggering algorithm (2), it can be easily seen that, for $t \in\left[t_{k} h+\tau_{k}, t_{k+1} h+\tau_{k+1}\right)$,

$$
e_{k}^{T}(t) \Omega e_{k}(t) \leq \rho x^{T}(t-\tau(t)) \Omega x(t-\tau(t)) .
$$

Utilizing $\tau(t)$ and $e_{k}(t)$, (5) can be rewritten as

$$
\begin{aligned}
\dot{x}(t)= & A x(t)+B \Xi K\left[x(t-\tau(t))+e_{k}(t)\right] \\
= & A x(t)+B \bar{\Xi} K x(t-\tau(t))+B \bar{\Xi} K e_{k}(t) \\
& +B(\Xi-\bar{\Xi}) K x(t-\tau(t))+B(\Xi-\bar{\Xi}) K e_{k}(t),
\end{aligned}
$$

where $t \in\left[t_{k} h+\tau_{k}, t_{k+1} h+\tau_{k+1}\right)$.

For the system (17), we supplement the initial condition of the state $x(t)$ on $\left[\tau_{M}, 0\right]$ as

$$
x(t)=\phi(t), \quad t \in\left[\tau_{M}, 0\right],
$$

where $\phi(t)$ is a continuous function on $\left[\tau_{M}, 0\right]$.

Remark 4. If $\tau_{k}=0$, it means that no transmission delay exists or transmission delay can be ignored, and the maximum sampling period is $\tau_{M}$. Note that $\tau_{M}=h+\bar{\tau}$. If $\tau_{k}>0$, the selecting sampling period $h<\tau_{M}, \bar{\tau}=\tau_{M}-h$, is the allowable maximum transmission delay.

Remark 5. Notice that if $\rho=0$, then $e_{k}(t)=0$; in this situation, the model (17) reduces to a model for a networked control system with a time-triggered scheme. It means that, if $\rho \rightarrow 0^{+}$, the dynamic of the system under eventtriggered scheme will approach to the one with a timetriggered scheme.

In the following, we will introduce the following definitions and lemmas, which are needed in the next section.

Definition 6. For a given function $V: C_{F_{0}}^{b}\left(\left[-\tau_{M}, 0\right], \mathbb{R}^{n}\right) \times S$, its infinitesimal operator $\mathscr{L}$ is defined as

$$
\mathscr{L}(V \eta(t))=\lim _{\Delta \rightarrow 0^{+}} \frac{1}{\Delta}\left[\mathbb{E}\left(V\left(\eta_{t}+\Delta\right) \mid \eta_{t}\right)-V\left(\eta_{t}\right)\right] .
$$

Definition 7. System (17) is said to be exponentially of mean square stability (EMSS) if there exist constants $\alpha>0$ and $\epsilon>$ 0 such that for $t \geq 0$,

$$
\mathbb{E}\left\{\|x(t)\|^{2}\right\} \leq \alpha e^{-\epsilon t} \mathbb{E}\left\{\sup _{-\tau_{M} \leq s \leq 0}\|\psi(s)\|^{2}\right\} .
$$

Lemma 8 (see [17]). For any vectors $x, y \in R^{n}$ and positive definite matrix $Q \in R^{n \times n}$, the following inequality holds:

$$
2 x^{T} y \leq x^{T} Q x+y^{T} Q^{-1} y .
$$

Lemma 9 (see [18]). $\Xi_{1}, \Xi_{2}$, and $\Omega$ are matrices with appropriate dimensions, $\tau(t)$ is a function of $t$, and $0 \leq \tau(t) \leq \tau_{M}$; then

$$
\tau(t) \Xi_{1}+\left(\tau_{M}-\tau(t)\right) \Xi_{2}+\Omega<0
$$

if and only if

$$
\begin{gathered}
\tau_{M} \Xi_{1}+\Omega<0, \\
\tau_{M} \Xi_{2}+\Omega<0 .
\end{gathered}
$$

\section{Main Results}

In this section, the following theorem provides the EMSS criteria for system (17) with the controller (4) under the event generator (2).

Theorem 10. For given $\mu_{i}, \sigma_{i}(i=1, \ldots, m), \rho$, and matrix $K$, the system described by (17) is EMSS, if there exists matrices $P>0, Q>0, R>0, \Omega>0$, and $N, M$ with which appropriate dimensions such that for $s=1,2$,

$$
\Sigma(s)=\left[\begin{array}{cccc}
\Sigma_{11}+\Gamma+\Gamma^{T} & * & * & * \\
\Sigma_{21} & -R & * & * \\
\Sigma_{31} & 0 & \Sigma_{33} & * \\
\Sigma_{41}(s) & 0 & 0 & -R
\end{array}\right]<0, \quad s=1,2,
$$

where

$$
\begin{aligned}
& \Sigma_{11}=\left[\begin{array}{cccc}
P A+A^{T} P+Q & * & * & * \\
K^{T} \bar{\Xi}^{T} B^{T} P & \rho \Omega & * & * \\
0 & 0 & -Q & * \\
K^{T} \bar{\Xi}^{T} B^{T} P & 0 & 0 & -\Omega
\end{array}\right], \\
& \Gamma=\left[\begin{array}{lll}
N & -N+M-M & 0
\end{array}\right], \\
& \Sigma_{21}=\left[\begin{array}{llll}
\sqrt{\tau_{M}} R A & \sqrt{\tau_{M}} R B \bar{\Xi} K & 0 & \sqrt{\tau_{M}} R B \bar{\Xi} K
\end{array}\right], \\
& \Sigma_{31}=\left[\begin{array}{cccc}
0 & \Pi & 0 & 0 \\
0 & 0 & 0 & \Pi
\end{array}\right], \\
& \Pi=\left[\begin{array}{c}
\sigma_{1} \sqrt{\tau_{M}} R B C_{1} K \\
\sigma_{2} \sqrt{\tau_{M}} R B C_{2} K \\
\vdots \\
\sigma_{m} \sqrt{\tau_{M}} R B C_{m} K
\end{array}\right], \\
& \Sigma_{33}=\operatorname{diag}\{\underbrace{-R, \ldots,-R}_{2 m}\} \text {, } \\
& \Sigma_{41}(1)=\sqrt{\tau_{M}} N^{T}, \quad \Sigma_{41}(2)=\sqrt{\tau_{M}} M^{T} .
\end{aligned}
$$

Proof. Choose the following Lyapunov functional candidate as

$$
V\left(x_{t}\right)=V_{1}\left(x_{t}\right)+V_{2}\left(x_{t}\right)+V_{3}\left(x_{t}\right) \text {, }
$$

where

$$
\begin{gathered}
V_{1}\left(x_{t}\right)=x^{T}(t) P x(t), \\
V_{2}\left(x_{t}\right)=\int_{t-\tau_{M}}^{t} x^{T}(s) Q x(s) d s \\
V_{3}\left(x_{t}\right)=\int_{t-\tau_{M}}^{t} \int_{s}^{t} \dot{x}^{T}(v) R \dot{x}(v) d v d s,
\end{gathered}
$$

in which $P, Q$, and $R$ are symmetric positive definite matrices. 
From the definition of $\Xi$, it can be concluded that $\mathbb{E}\{\Xi-$ $\bar{\Xi}\}=\operatorname{diag}\{0, \ldots, 0\}$, and using the infinitesimal operator (19) for $V\left(x_{t}\right)$ and taking expectation on it, we obtain

$$
\begin{gathered}
\mathbb{E}\left\{\mathscr{L} V_{1}\left(x_{t}\right)\right\}=2 x^{T}(t) P Y(t), \\
\mathbb{E}\left\{\mathscr{L} V_{2}\left(x_{t}\right)\right\}=x^{T}(t) Q x(t)-x^{T}(t-\tau(t)) Q x(t-\tau(t)), \\
\mathbb{E}\left\{\mathscr{L} V_{3}\left(x_{t}\right)\right\}=\mathbb{E}\left[\tau_{M} \dot{x}^{T}(t) R \dot{x}(t)\right]-\int_{t-\tau_{M}}^{t} \dot{x}^{T}(s) R \dot{x}(s) d s,
\end{gathered}
$$

where $\Upsilon(t)=A x(t)+B \bar{\Xi} K x(t-\tau(t))+B \bar{\Xi} K e_{k}(t)$.

Notice that

$$
\begin{aligned}
\mathbb{E}\left[\tau_{M} \dot{x}^{T}(t) R \dot{x}(t)\right]= & \tau_{M} \Upsilon^{T}(t) R \Upsilon(t) \\
& +\sum_{i=1}^{m} \tau_{M} \sigma_{i}^{2} e_{k}^{T}(t) K^{T} C_{i}^{T} B^{T} R B C_{i} K e_{k}(t) \\
& +\sum_{i=1}^{m} \tau_{M} \sigma_{i}^{2} x^{T}(t-\tau(t)) K^{T} C_{i}^{T} B^{T} R B C_{i} \\
& \times K x(t-\tau(t)) .
\end{aligned}
$$

Combining (28) and (29), we obtain

$$
\begin{aligned}
\mathbb{E}\left\{\mathscr{L} V\left(x_{t}\right)\right\}= & 2 x^{T}(t) P \Upsilon(t)+x^{T}(t) Q x(t) \\
& -x^{T}(t-\tau(t)) Q x(t-\tau(t)) \\
& +\tau_{M} \Upsilon^{T}(t) R \Upsilon(t) \\
& +\sum_{i=1}^{m} \tau_{M} \sigma_{i}^{2} e_{k}^{T}(t) K^{T} C_{i}^{T} B^{T} R B C_{i} K e_{k}(t) \\
& +\sum_{i=1}^{m} \tau_{M} \sigma_{i}^{2} x^{T}(t-\tau(t)) K^{T} C_{i}^{T} B^{T} R B C_{i} \\
& -\int_{t-\tau_{M}}^{t} \dot{x}^{T}(s) R \dot{x}(s) d s+\Gamma_{1}+\Gamma_{2},
\end{aligned}
$$

where $\Gamma_{1}$ and $\Gamma_{2}$ are introduced by employing free weight matrix method $[19,20]$

$$
\begin{gathered}
\Gamma_{1}=2 \zeta^{T}(t) N\left[x(t)-x(t-\tau(t))-\int_{t-\tau(t)}^{t} \dot{x}(s) d s\right]=0, \\
\Gamma_{2}=2 \eta^{T}(t) M\left[x(t-\tau(t))-x\left(t-\tau_{M}\right)-\int_{t-\tau_{M}}^{t-\tau(t)} \dot{x}(s) d s\right]=0,
\end{gathered}
$$

where $N$ and $M$ are matrices with appropriate dimensions, and

$$
\zeta^{T}(t)=\left[x^{T}(t) x^{T}(t-\tau(t)) x^{T}\left(t-\tau_{M}\right) e_{k}^{T}(t)\right] .
$$

By Lemma 8, we have

$$
\begin{aligned}
-2 \zeta^{T}(t) N \int_{t-\tau(t)}^{t} \dot{x}(s) d s \leq & \tau(t) \zeta^{T}(t) N R^{-1} N^{T} \zeta(t) \\
& +\int_{t-\tau(t)}^{t} \dot{x}^{T}(s) R \dot{x}(s) d s, \\
-2 \zeta^{T}(t) M \int_{t-\tau_{M}}^{t-\tau(t)} \dot{x}(s) d s \leq & \left(\tau_{M}-\tau(t)\right) \zeta^{T}(t) N R^{-1} M^{T} \zeta(t) \\
& +\int_{t-\tau_{M}}^{t-\tau(t)} \dot{x}^{T}(s) R \dot{x}(s) d s .
\end{aligned}
$$
that

Combining (16), substitute (33) into (30), and we obtain

$$
\begin{aligned}
\mathbb{E}\left\{\mathscr{L} V\left(x_{t}\right)\right\} \leq & 2 x^{T}(t) P \Upsilon(t)+x^{T}(t) Q x(t) \\
& -x^{T}(t-\tau(t)) Q x(t-\tau(t)) \\
& +\tau_{M} \Upsilon^{T}(t) R \Upsilon(t) \\
& +\sum_{i=1}^{m} \tau_{M} \sigma_{i}^{2} e_{k}^{T}(t) K^{T} C_{i}^{T} B^{T} R B C_{i} K e_{k}(t) \\
& +\sum_{i=1}^{m} \tau_{M} \sigma_{i}^{2} x^{T}(t-\tau(t)) K^{T} C_{i}^{T} B^{T} R B C_{i} \\
& \quad \times K x(t-\tau(t)) \\
& +2 \zeta^{T}(t) N[x(t)-x(t-\tau(t))]+2 \zeta^{T}(t) M \\
& \times\left[x(t-\tau(t))-x\left(t-\tau_{M}\right)\right] \\
& -e_{k}^{T}(t) \Omega e_{k}(t)+\rho x^{T}(t-\tau(t)) \Omega x(t-\tau(t)) \\
& +\left(\tau_{M}-\tau(t)\right) \zeta^{T}(t) M R^{-1} M^{T} \zeta(t) \\
& +\tau(t) \zeta^{T}(t) N R^{-1} N^{T} \zeta(t) ;
\end{aligned}
$$

that is,

$$
\mathbb{E}\left\{\mathscr{L} V\left(x_{t}\right)\right\} \leq \zeta^{T}(t) \Theta \zeta(t),
$$

where $\Theta=\Sigma_{11}+\Gamma+\Gamma^{T}+\Sigma_{21}^{T} R^{-1} \Sigma_{21}-\Sigma_{31}^{T} \Sigma_{31}^{-1} \Sigma_{31}+\left(\tau_{M}-\right.$ $\tau(t)) M R^{-1} M^{T}+\tau(t) N R^{-1} N^{T}$.

By using Schur complement and Lemma 9, we have $\Theta<$ 0 , if and only if the following holds:

$$
\begin{array}{r}
{\left[\begin{array}{cc}
\Sigma_{11}+\Gamma+\Gamma^{T}+\sum_{21}^{T} R^{-1} \Sigma_{21}-\Sigma_{31}^{T} \Sigma_{31}^{-1} \Sigma_{31} & * \\
\sum_{41}(s) & -R
\end{array}\right]<0} \\
s=1,2 .
\end{array}
$$

By using Schur complement, we can obtain that (36) is equivalent to (24). Furthermore,

$$
\mathbb{E}\left\{\mathscr{L} V\left(x_{t}\right)\right\} \leq-\lambda \zeta^{T}(t) \zeta(t),
$$


where $\lambda=\min \left\{\lambda_{\min }[\Theta]\right\}$. Define a new function as

$$
W\left(x_{t}\right)=e^{\epsilon t} V\left(x_{t}\right)
$$

Its infinitesimal operator $\mathscr{L}$ is given by

$$
\mathscr{L} W\left(x_{t}\right)=\epsilon e^{\epsilon t} V\left(x_{t}\right)+e^{\epsilon t} \mathscr{L} V\left(x_{t}\right) .
$$

From (39), we can obtain that

$$
\begin{aligned}
\mathbb{E} W\left(x_{t}\right)-\mathbb{E} W\left(x_{0}\right)= & \int_{0}^{t} \epsilon e^{\epsilon s} \mathbb{E}\left\{V\left(x_{s}\right)\right\} \\
& +\int_{0}^{t} e^{\epsilon s} \mathbb{E}\left\{\mathscr{L} V\left(x_{s}\right)\right\} .
\end{aligned}
$$

Then using the similar method of [15], we can observe that there exists a positive number $\alpha$ such that for $t \geq 0$

$$
\mathbb{E}\left\{V\left(x_{t}\right)\right\} \leq \alpha \sup _{-\tau_{M} \leq s \leq 0} e^{-\epsilon s} \mathbb{E}\left\{\|\psi(s)\|^{2}\right\} .
$$

Since $V\left(x_{t}\right) \geq \lambda_{\min }(P) x^{T}(t) x(t)$, it can be shown from (41) that, for $t \geq 0$,

$$
\mathbb{E}\left\{x^{T}(t) x(t)\right\} \leq \bar{\alpha} e^{-\epsilon t} \sup _{-\tau_{M} \leq s \leq 0} \mathbb{E}\left\{\|\psi(s)\|^{2}\right\},
$$

where $\bar{\alpha}=\alpha / \lambda_{\min }(P)$. The proof can be completed.

In the following, based on Theorem 10, we will design the feedback gain $K$ in (4) under the event-trigger (2).

Theorem 11. For given $\mu_{i}, \sigma_{i}(i=1, \ldots, m), \rho$ and $\varepsilon$, system (17) with the feedback gain $K=Y X^{-1}$ under the event trigger condition (2) is EMSS if there exist matrices $X>0, \widetilde{Q}>0$, $\widetilde{R}>0, \widetilde{\Omega}>0$, and $\widetilde{N}, \widetilde{M}$ with appropriate dimensions such that $s=1,2$

$$
\left[\begin{array}{cccc}
\widetilde{\Sigma}_{11}+\widetilde{\Gamma}+\widetilde{\Gamma}^{T} & * & * & * \\
\widetilde{\Sigma}_{21} & -2 \varepsilon X+\varepsilon^{2} \widetilde{R} & * & * \\
\widetilde{\Sigma}_{31} & 0 & \widetilde{\Sigma}_{33} & * \\
\widetilde{\Sigma}_{41}(s) & 0 & 0 & -2 \varepsilon X+\varepsilon^{2} \widetilde{R}
\end{array}\right]<0,
$$$$
s=1,2 \text {, }
$$

where

$$
\begin{gathered}
\widetilde{\Sigma}_{11}=\left[\begin{array}{cccc}
A X+X A^{T}+\widetilde{Q} & * & * & * \\
Y^{T} \bar{\Xi}^{T} B^{T} & \rho \widetilde{\Omega} & * & * \\
0 & 0 & -\widetilde{Q} & * \\
Y^{T} \bar{\Xi}^{T} B^{T} & 0 & 0 & -\widetilde{\Omega}
\end{array}\right], \\
\widetilde{\Gamma}=\left[\begin{array}{llll}
\widetilde{N} & -\widetilde{N}+\widetilde{M} & -\widetilde{M} & 0
\end{array}\right], \\
\widetilde{\Sigma}_{21}=\left[\begin{array}{llll}
\sqrt{\tau_{M}} A X & \sqrt{\tau_{M}} B \bar{\Xi} Y & 0 & \sqrt{\tau_{M}} B \bar{\Xi} Y
\end{array}\right],
\end{gathered}
$$

$$
\begin{gathered}
\widetilde{\Sigma}_{31}=\left[\begin{array}{cccc}
0 & \widetilde{\Pi} & 0 & 0 \\
0 & 0 & 0 & \widetilde{\Pi}
\end{array}\right], \quad \widetilde{\Pi}=\left[\begin{array}{c}
\sigma_{1} \sqrt{\tau_{M}} B C_{1} Y \\
\sigma_{2} \sqrt{\tau_{M}} B C_{2} Y \\
\vdots \\
\sigma_{m} \sqrt{\tau_{M}} B C_{m} Y
\end{array}\right], \\
\widetilde{\Sigma}_{33}=\operatorname{diag}\{\underbrace{-2 \varepsilon X+\varepsilon^{2} \widetilde{R}, \ldots,-2 \varepsilon X+\varepsilon^{2} \widetilde{R}}_{2 m}\}, \\
\widetilde{\Sigma}_{41}(1)=\sqrt{\tau_{M}} \widetilde{N}^{T}, \quad \widetilde{\Sigma}_{41}(2)=\sqrt{\tau_{M}} \widetilde{M}^{T} .
\end{gathered}
$$

Proof. By using Schur complement, we can obtain that the following is equivalent to (24):

$$
\left[\begin{array}{cccc}
\Sigma_{11}+\Gamma+\Gamma^{T} & * & * & * \\
\Phi_{21} & -P R^{-1} P & * & * \\
\Phi_{31} & 0 & \Phi_{33} & * \\
\Sigma_{41}(s) & 0 & 0 & -R
\end{array}\right]<0, \quad s=1,2
$$

where

$$
\begin{aligned}
& \Phi_{21}=\left[\begin{array}{llll}
\sqrt{\tau_{M}} P A & \sqrt{\tau_{M}} P B \bar{\Xi} K & 0 & \sqrt{\tau_{M}} P B \bar{\Xi} K
\end{array}\right], \\
& \Phi_{31}=\left[\begin{array}{cccc}
0 & \Pi^{1} & 0 & 0 \\
0 & 0 & 0 & \Pi^{1}
\end{array}\right], \quad \Pi^{1}=\left[\begin{array}{c}
\sigma_{1} \sqrt{\tau_{M}} P B C_{1} K \\
\sigma_{2} \sqrt{\tau_{M}} P B C_{2} K \\
\vdots \\
\sigma_{m} \sqrt{\tau_{M}} P B C_{m} K
\end{array}\right] \text {, } \\
& \Phi_{33}=\operatorname{diag}\{\underbrace{-P R^{-1} P, \ldots,-P R^{-1} P}_{2 m}\} \text {. }
\end{aligned}
$$

Due to $\left(R-\varepsilon^{-1} P\right) R^{-1}\left(R-\varepsilon^{-1} P\right) \geq 0$, we have

$$
-P R^{-1} P \leq-2 \varepsilon P+\varepsilon^{2} R .
$$

Substituting $-P R^{-1} P$ with $-2 \varepsilon P+\varepsilon^{2} R$ into (45), we obtain

$$
\left[\begin{array}{cccc}
\Sigma_{11}+\Gamma+\Gamma^{T} & * & * & * \\
\Phi_{21} & -2 \varepsilon P+\varepsilon^{2} R & * & * \\
\Phi_{31} & 0 & \widehat{\Phi}_{33} & * \\
\Sigma_{41}(s) & 0 & 0 & -R
\end{array}\right]<0, \quad s=1,2
$$

where $\widehat{\Phi}_{33}=\operatorname{diag}\{\underbrace{-2 \varepsilon P+\varepsilon^{2} R, \ldots,-2 \varepsilon P+\varepsilon^{2} R}_{2 m}\}$.

Denoting $X=P^{-1}, \widetilde{Q}=X Q X, \widetilde{R}=X R X, \widetilde{N}=$ $X N X, \widetilde{M}=X M X, \widetilde{\Omega}=X \Omega X$, and $Y=K X$, then preand postmultiplying (48) with $\operatorname{diag}\{\underbrace{X, \ldots, X}_{2 m+5}, I\},(43)$ can be obtained.

Remark 12. Theorem 11 shows that, for given $\rho$ and $\varepsilon$, we can obtain the feedback gain $K$ by solving a set of LMIs in (43); on the other hand, using Theorem 11, for the preselected $\Omega$ and the feedback gain $K$, event-triggered parameter $\rho$ can be obtained. Therefore, we can use Theorem 11 to codesign the the feedback gain $K$ and the event-triggered parameter. 
Remark 13. Notice that (43) includes the information transmission delay, and we can use (43) to obtain the feedback gain and the event-triggered parameter, which can be used to guarantee the required performance even though the transmission delay exists.

\section{Simulation Examples}

Example 14. To illustrate the effectiveness and application of the proposed method, we consider an inverted pendulum on top of a moving cart. The plants linearized state is depicted as the following system [21]:

$$
\dot{x}(t)=\left[\begin{array}{cccc}
0 & 1 & 0 & 0 \\
0 & 0 & \frac{-m g}{M} & 0 \\
0 & 0 & 0 & 1 \\
0 & 0 & \frac{g}{l} & 0
\end{array}\right] x(t)+\left[\begin{array}{c}
0 \\
\frac{1}{M} \\
0 \\
\frac{-1}{M l}
\end{array}\right] u(t) \text {, }
$$

where $x^{T}=\left[\begin{array}{llll}x_{1} & x_{2} & x_{3} & x_{4}\end{array}\right]=\left[\begin{array}{llll}y & \dot{y} & \theta & \dot{\theta}\end{array}\right]$ is the system state, $y$ is the carts position, and $\theta$ is the pendulum bob's angle with respect to the vertical. $M=10 \mathrm{~kg}$ is the cart mass, $m=1 \mathrm{~kg}$ is the mass of the pendulum bob, $l=3 \mathrm{~m}$ is the length of the pendulum arm, and $g=10 \mathrm{~m} / \mathrm{s}^{2}$ is gravitational acceleration. The initial state is the vector $x_{0}=\left[\begin{array}{llll}0.98 & 0 & 0.2 & 0\end{array}\right]$.

In the following, we will give two cases. Case 1 is used to show how the upper bound of $\tau_{M}$ varies along the values of $\rho$, under given feedback gain $K$ as (50), when there is no failure in the actuator. In Case 2, we consider that the actuators have probabilistic failure rates; firstly, we give an example to design both the feedback and the trigger parameters, and the upper bound of $\tau_{M}$ and the release interval for $t \in[o, 40]$ are also derived; secondly, we suppose that the feedback gain $K$ is given as (50) and study how the upper bound of $\tau_{M}$ varies along the values of $\rho$.

Case 1. When $\Xi=1$, that is, there is no failure in the actuator, let

$$
K=\left[\begin{array}{llll}
2 & 12 & 378 & 210
\end{array}\right]
$$

which is the same as the one in [22].

For given $\sigma_{1}=0, \varepsilon=0.53$, and $\rho=0.2$, by using Theorem 10, we can have $\tau_{M}=0.1270$ and

$$
\Omega=\left[\begin{array}{llll}
0.0001 & 0.0003 & 0.0112 & 0.0062 \\
0.0003 & 0.0023 & 0.0666 & 0.0376 \\
0.0112 & 0.0666 & 2.1100 & 1.1662 \\
0.0062 & 0.0376 & 1.1662 & 0.6510
\end{array}\right]
$$

Also, we can obtain Table 1 and Figure 2, which describe the upper bound of $\tau_{M}$ varying along the values of $\rho$ and the state response of (17).

With feedback gain $K$ as (50), from Table 1, we can see that when $\rho=0.53$ and $\sigma=0.2$, the upper bound of $\tau_{M}$ is 0.1270 . Suppose that $\tau_{k}=0$; since $\tau_{M}=h+\bar{\tau}$, it can be known that the maximum sampling period is 0.1270 . Moreover, under our event-trigger scheme, the maximum release period is 2.3 .

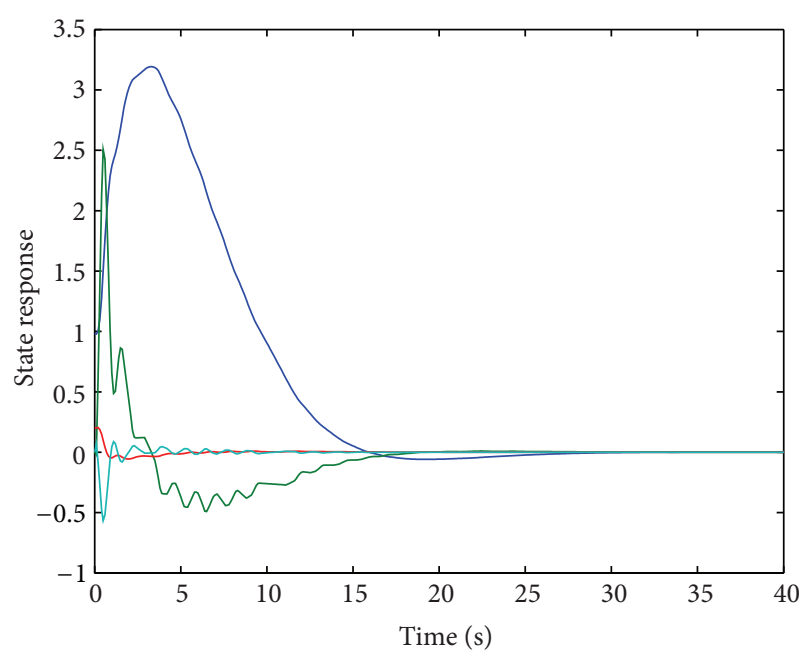

FIGURE 2: The state response of (49) with the feedback gain (50) for given $\Xi=1, \sigma_{1}=0, \varepsilon=0.53$, and $\rho=0.2$.

TABLE 1: The computation results of the upper bound $\tau_{M}$ for given $\bar{\Xi}=1, \sigma=0$ and $\varepsilon=0.53$.

\begin{tabular}{lccccc}
\hline$\rho$ & 0 & 0.05 & 0.1 & 0.15 & 0.2 \\
\hline The upper bound of $\tau_{M}$ & 0.1987 & 0.1582 & 0.1445 & 0.1349 & 0.1270 \\
\hline
\end{tabular}

It can be seen that under the same conditions, our scheme can provide a larger release interval than some existing ones in $[21,22]$.

Case 2. When $\bar{\Xi}=0.6$ and $\sigma_{1}=0.02$, that is, the actuators have probabilistic failure rate, setting $\varepsilon=0.53$ and $\rho=0.2$, by using Theorem 11, we can obtain the feedback gain $K$ of (4) as follows:

$$
\begin{gathered}
K=\left[\begin{array}{llll}
5.0696 & 23.2085 & 594.9876 & 331.9409
\end{array}\right] \\
\Omega=\left[\begin{array}{cccc}
162.1817 & 72.523 & -13.8855 & -11.9519 \\
72.5232 & 525.4131 & -67.738 & -32.7170 \\
-13.8855 & -67.7382 & 52.8001 & -70.5906 \\
-11.9519 & -32.7170 & -70.5906 & 132.2203
\end{array}\right] .
\end{gathered}
$$

For given $\bar{\Xi}=0.6, \sigma=0.02$, and $\varepsilon=0.53$, under different values of $\rho$, the upper bound of $\tau_{M}$ and the release interval for $t \in[0,40]$ are given in Table 2 . Figures 3 and 4 , respectively, represent the release instants and release interval and the probabilistic actuator failures, when $\bar{\Xi}=0.6, \sigma=0.02$, $\varepsilon=0.53, \rho=0.1$, and $\tau_{M}=0.2399$.

When $K$ is given as (50), let $\bar{\Xi}=0.6, \bar{\Xi}=1, \sigma_{1}=0$, and $\varepsilon=0.53$, and the upper bound of $\tau_{M}$ varies along the values of $\rho$, which is shown in Table 3 .

Remark 15. For given $\rho$ and $\varepsilon$, we can obtain the upper bound of $\tau_{M}$. we can see that the larger $\rho$, the larger average release period; Thus, the load of network communication delay is reduced and the transmission delay is decreased. This fact can be illustrated by Tables 1, 2 and 3 . 


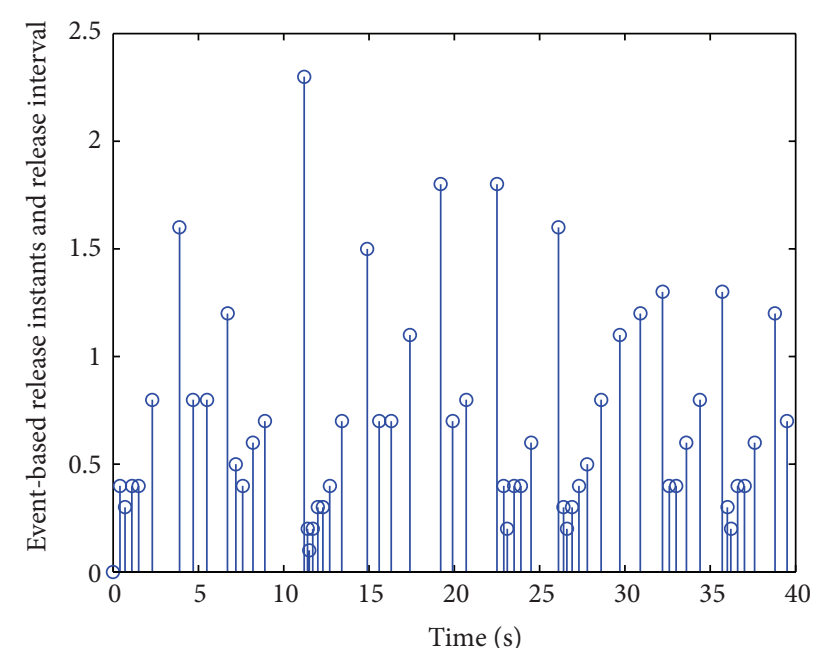

FIgURE 3: The release instants and release interval for given $\bar{\Xi}=0.6$, $\sigma=0.02$, and $\varepsilon=0.53$.

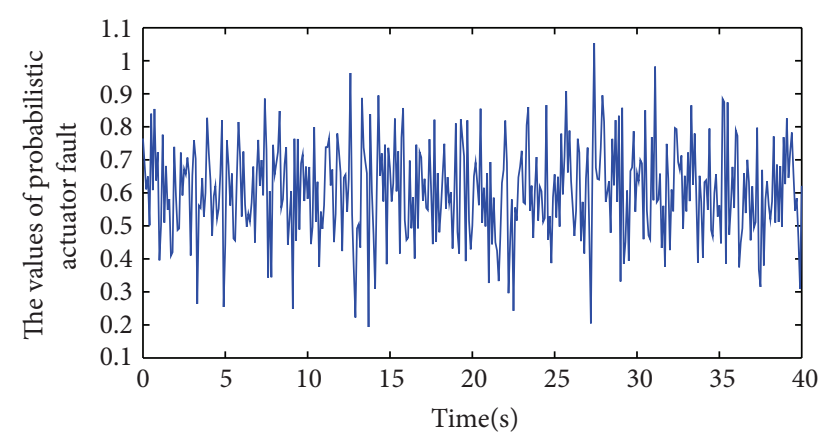

FIgURE 4: The probabilistic actuator failures.

TABLE 2: Some computation results for given $\bar{\Xi}=0.6, \sigma=0.02$, and $\varepsilon=0.53$.

\begin{tabular}{lccccc}
\hline$\rho$ & 0 & 0.1 & 0.2 & 0.3 & 0.4 \\
\hline $\begin{array}{l}\text { The upper bound of } \\
\tau_{M}\end{array}$ & 0.3556 & 0.2399 & 0.1789 & 0.1394 & 0.1085 \\
$\begin{array}{l}\text { The maximum release } \\
\text { interval for } t \in[0,40]\end{array}$ & 0.1 & 2.3 & 1.1 & 1.2 & 2.3 \\
$\begin{array}{l}\text { The total trigger times } \\
\text { for } t \in[0,40]\end{array}$ & 401 & 57 & 78 & 111 & 122 \\
\hline
\end{tabular}

TABLE 3: The computation results of the upper bound $\tau_{M}$ for given $\bar{\Xi}=0.6, \sigma=0.02$, and $\varepsilon=0.53$.

\begin{tabular}{lccccc}
\hline$\rho$ & 0 & 0.05 & 0.1 & 0.15 & 0.2 \\
\hline The upper bound of $\tau_{M}$ & 0.2963 & 0.2197 & 0.1479 & 0.0546 & 0 \\
\hline
\end{tabular}

\section{Conclusion}

In order to save the communication network bandwidth, a novel event triggering scheme is used to determine when to transmit the sampled state information. Under this eventtriggered scheme, this paper considers networked systems with probabilistic actuators faults. In terms of different failure rates and the measurements distortion of every actuator, a new probabilistic actuator fault model for event-triggered networked control systems is proposed. By using Lyapunov functional method, criteria for the EMSS and criteria for codesigning both the feedback and the trigger parameters are derived in the form of linear matrix inequalities. A simulation example is given to illustrate that our event-triggered scheme can lead to a larger release period than some existing works.

\section{Acknowledgments}

This work is partly supported by the National Natural Science Foundation of China (No. 11226240), the Natural Science Foundation of Jiangsu Province of China (no. BK2012469), the Natural Science Foundation of the Jiangsu Higher Education Institutions of China (no. 12 KJD120001), the Young Teacher Supporting Foundation of Shanghai (no. ZZGJD12036), and a project funded by the Priority Academic Program Development of Jiangsu Higher Education Institutions (PAPD).

\section{References}

[1] J. P. Hespanha, P. Naghshtabrizi, and Y. Xu, "A survey of recent results in networked control systems," Proceedings of the IEEE, vol. 95, no. 1, pp. 138-172, 2007.

[2] T. Yang, "Networked control system: a brief survey," IEE Proceedings Control Theory and Applications, vol. 153, no. 4, pp. 403-412, 2006.

[3] Q.-L. Han, "A discrete delay decomposition approach to stability of linear retarded and neutral systems," Automatica, vol. 45, no. 2, pp. 517-524, 2009.

[4] H. Gao, X. Meng, and T. Chen, "Stabilization of networked control systems with a new delay characterization," IEEE Transactions on Automatic Control, vol. 53, no. 9, pp. 2142-2148, 2008.

[5] X. Wang and M. D. Lemmon, "Event-triggering in distributed networked control systems," IEEE Transactions on Automatic Control, vol. 56, no. 3, pp. 586-601, 2011.

[6] P. Tabuada, "Event-triggered real-time scheduling of stabilizing control tasks," IEEE Transactions on Automatic Control, vol. 52, no. 9, pp. 1680-1685, 2007.

[7] V. Nguyen and Y. Suh, "Networked estimation for event-based sampling systems with packet dropouts," Sensors, vol. 9, no. 4, pp. 3078-3089, 2009.

[8] A. Eqtami, D. V. Dimarogonas, and K. J. Kyriakopoulos, "Eventtriggered control for discrete-time systems," in Proceedings of the American Control Conference (ACC '10), pp. 4719-4724, July 2010.

[9] X. Wang and M. Lemmon, "On event design in event-triggered feedback systems," Automatica, vol. 47, no. 10, pp. 2319-2322, 2011.

[10] D. Yue, J. Lam, and D. Ho, "Reliable $H_{\infty}$ control of uncertain descriptor systems with multiple time delays," IEE Proceedings Control Theory and Applications, vol. 150, no. 6, pp. 557-564, 2003.

[11] D. Ye and G.-H. Yang, "Delay-dependent adaptive reliable $H_{\infty}$ control of linear time-varying delay systems," International Journal of Robust and Nonlinear Control, vol. 19, no. 4, pp. 462479, 2009. 
[12] E. Tian, D. Yue, and C. Peng, "Reliable control for networked control systems with probabilistic sensors and actuators faults," IET Control Theory \& Applications, vol. 4, no. 8, pp. 1478-1488, 2010.

[13] E. Tian, D. Yue, and C. Peng, "Reliable control for networked control systems with probabilistic actuator fault and random delays," Journal of the Franklin Institute, vol. 347, no. 10, pp. 19071926, 2010.

[14] F. O. Hounkpevi and E. E. Yaz, "Robust minimum variance linear state estimators for multiple sensors with different failure rates," Automatica, vol. 43, no. 7, pp. 1274-1280, 2007.

[15] X. He, Z. Wang, and D. Zhou, "Robust filtering for time-delay systems with probabilistic sensor faults," IEEE Signal Processing Letters, vol. 16, no. 5, pp. 442-445, 2009.

[16] G. Wei, Z. Wang, and H. Shu, "Robust filtering with stochastic nonlinearities and multiple missing measurements," Automatica, vol. 45, no. 3, pp. 836-841, 2009.

[17] Y. Y. Wang, L. Xie, and C. E. de Souza, "Robust control of a class of uncertain nonlinear systems," Systems \& Control Letters, vol. 19, no. 2, pp. 139-149, 1992.

[18] E. Tian, D. Yue, and Y. Zhang, "Delay-dependent robust $H_{\infty}$ control for T-S fuzzy system with interval time-varying delay," Fuzzy Sets and Systems, vol. 160, no. 12, pp. 1708-1719, 2009.

[19] D. Yue, Q.-L. Han, and J. Lam, "Network-based robust $H_{\infty}$ control of systems with uncertainty," Automatica, vol. 41, no. 6 , pp. 999-1007, 2005.

[20] Y. He, M. Wu, J.-H. She, and G.-P. Liu, "Parameter-dependent Lyapunov functional for stability of time-delay systems with polytopic-type uncertainties," IEEE Transactions on Automatic Control, vol. 49, no. 5, pp. 828-832, 2004.

[21] X. Wang, Event-triggering in cyber-physical systems [Ph.D. dissertation], University of Notre Dame, 2009.

[22] X. Wang and M. D. Lemmon, "Self-triggered feedback control systems with finite-gain $\mathscr{L}_{2}$ stability," IEEE Transactions on Automatic Control, vol. 54, no. 3, pp. 452-467, 2009. 


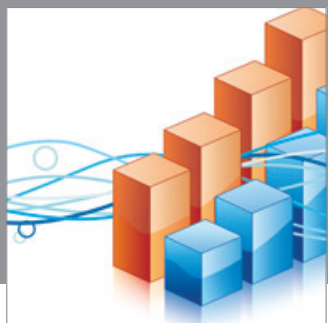

Advances in

Operations Research

mansans

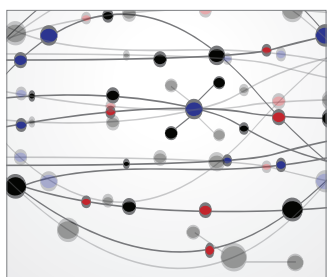

The Scientific World Journal
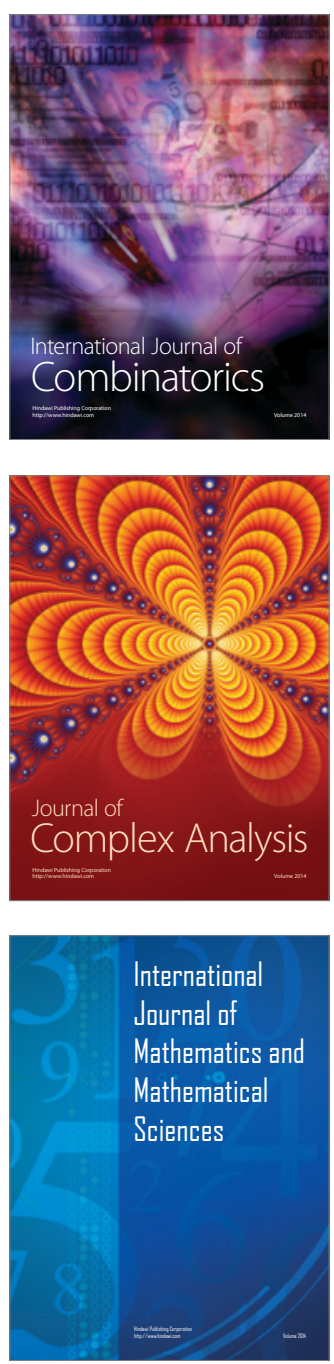
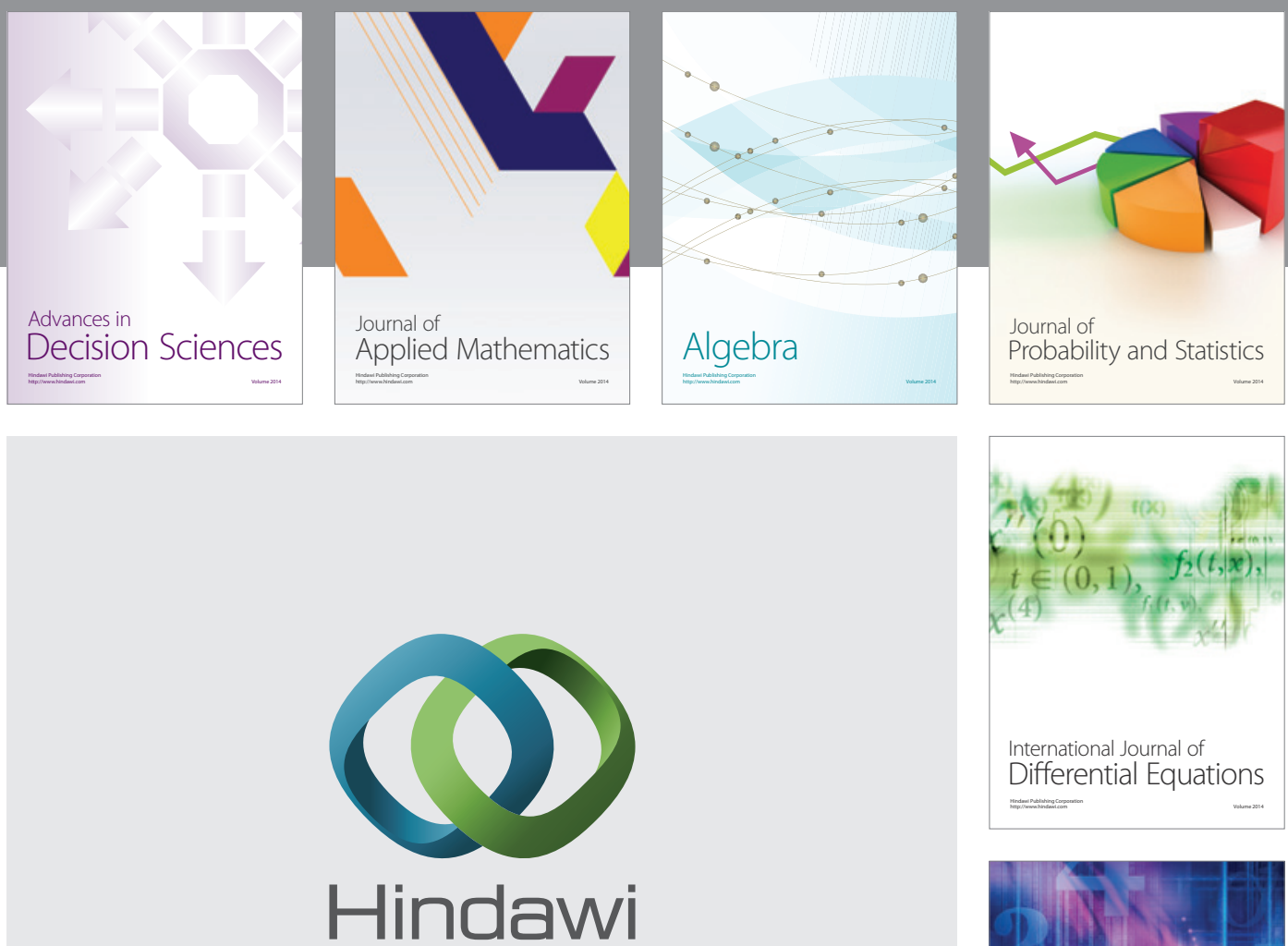

Submit your manuscripts at http://www.hindawi.com
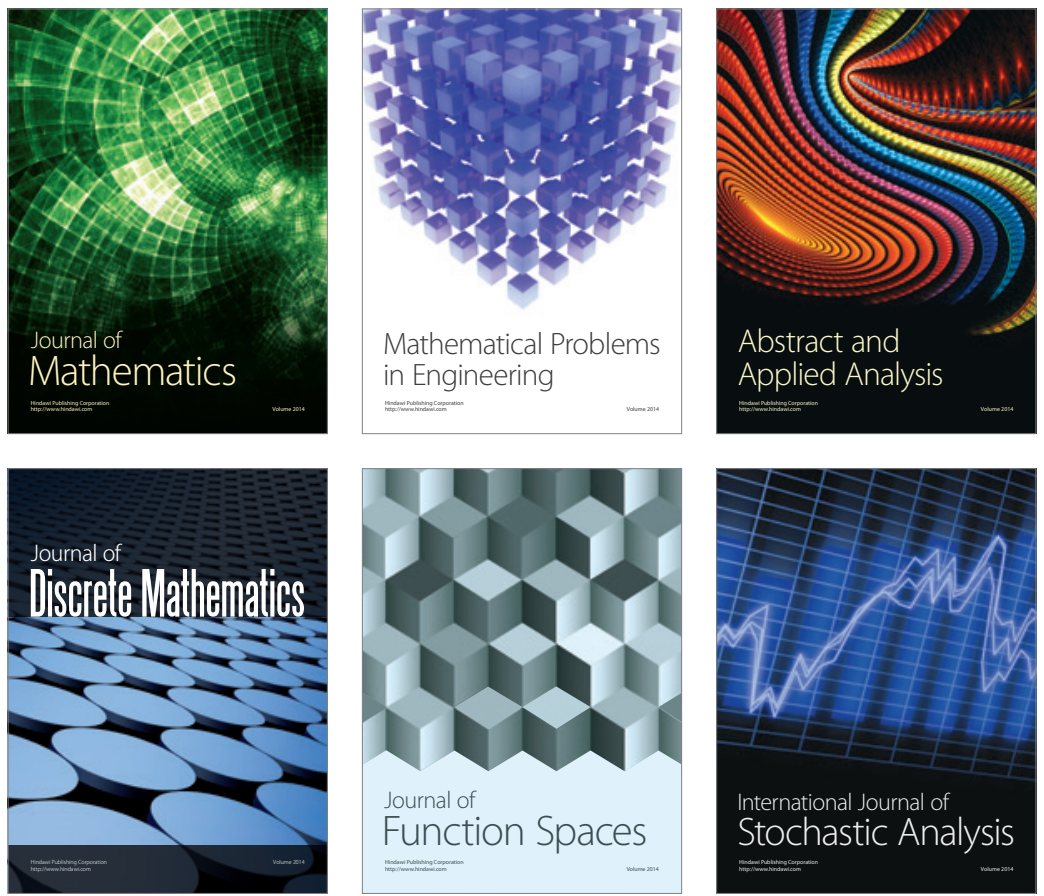

Journal of

Function Spaces

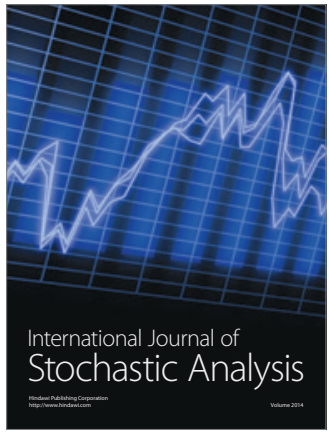

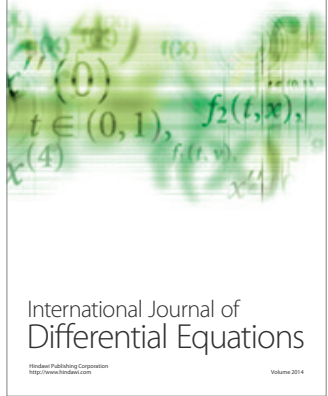
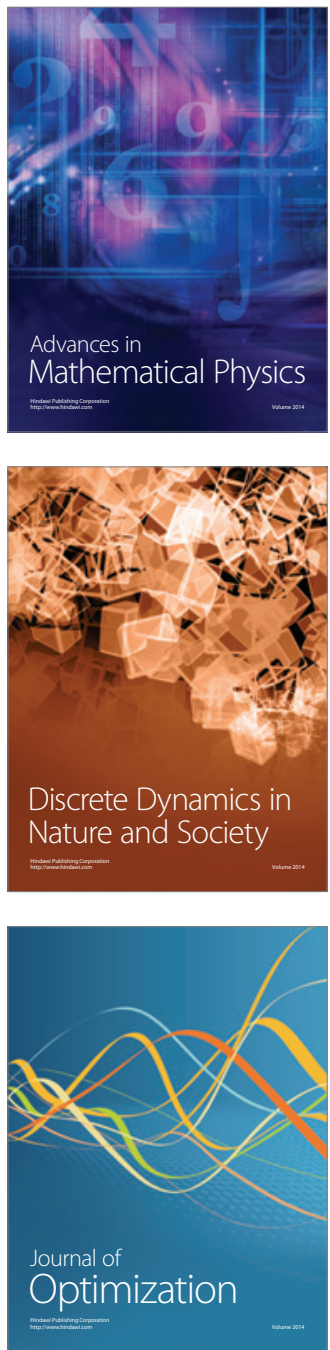